\title{
Our Supermassive Black Hole Rivaled the Sun in the Ancient X-ray Sky
}

\author{
Pau Amaro-Seoane \\ Institut de Ciències de l'Espai (CSIC-IEEC) at Campus UAB, \\ Carrer de Can Magrans s/n 08193 Barcelona, Spain \\ Kavli Institute for Astronomy and Astrophysics, Beijing 100871, China \\ Institute of Applied Mathematics, Academy of Mathematics and Systems Science, \\ CAS, Beijing 100190, China \\ Zentrum für Astronomie und Astrophysik, TU Berlin, \\ Hardenbergstraße 36, 10623 Berlin, Germany \\ Xian Chen $^{1}$ \\ Astronomy Department, School of Physics, Peking University, 100871 Beijing, China \\ Kavli Institute for Astronomy and Astrophysics, Beijing 100871, China
}

\begin{abstract}
Sagittarius A* $\left(\mathrm{SgrA}^{*}\right)$ lying in the Galactic Centre $8 \mathrm{kpc}$ from Earth, hosts the closest supermassive black hole known to us. It is now inactive, but there is evidence indicating that about six million years ago it underwent a powerful outburst where the luminosity could have approached the Eddington limit. Motivated by the fact that in extragalaxies the supermassive black holes with similar masses and near-Eddington luminosities are usually strong X-ray emitters, we calculate here the X-ray luminosity of SgrA*. For that, we assume that the outburst was due to accretion of gas or the tidal disruption of a star. We show that these cases could precipitate on Earth a hard X-ray (i.e. $h \nu>2 \mathrm{keV}$ ) flux comparable to that from the current quiescent sun. The flux in harder energy band $20 \mathrm{keV}<h \nu<100 \mathrm{keV}$, however, surpasses that from an X-class solar flare, and the irradiation timescale is also much longer, ranging from weeks to $10^{5}$ years depending on the outburst scenario. In the solar system gas giants will suffer the biggest impact in their atmospheres. Lower-mass planets such as
\end{abstract}

\footnotetext{
${ }^{1}$ Corresponding author.
} 
Earth receive a level of radiation that might have played a role in the evolution of their primitive atmospheres, so that a detailed study of the consequences deserves further investigation. Planetary systems closer to SgrA* receive higher irradiance levels, making them more likely uninhabitable.

Keywords: Galactic Centre; Supermassive Black Holes; High-Energy Astrophysics; Solar System

\section{Introduction}

Supermassive black holes (SMBHs) intermittently undergo electromagnetic outbursts at the expense of the gravitational energies of the infalling gas or stars, but during most of their lifetime they are quiescent, "inactive", and are found in the nuclei of galaxies [1]. Our own Galaxy harbours one, SgrA*, with a mass of about $4 \times 10^{6} M_{\odot}$ [see the review of 2 , and references therein].

Although it is accepted that at early ages our Milky Way (MW) was an Active Galactic Nucleus (AGN), the possibility that during the past few Myrs SgrA* had an activity in the range of an AGN has not been addressed because, at least partly, AGNs have been thought to be uncommon. The common believe was that AGNs are mostly triggered via galaxy mergers. There is evidence suggesting that our Galaxy has not experienced a major merger during the past 10 billion years [3]. This led to believe that the MW could not have had such an activity recently, because the merger timescale is considered to be too long.

However, this situation has changed significantly. Recent studies of AGNs in the local universe suggest that SMBHs less massive than $10^{7} M_{\odot}$, like $\mathrm{Sgr} A^{*}$, could be frequently activated by stochastic processes such as gravitational instability [4]. The frequency could be as high as once every $10^{7}-10^{8}$ years, with each burst lasting about $10^{5}$ years [4]. Because of them, it is not ruled out that the Galactic Centre could have had a significant activity at some point during the past 10 Myrs.

On the observational front, there is (mounting) observational evidence that SgrA* could have undergone an energetic outburst about $2-8$ Myr ago [5, 
6, 7, 8, 9, 10], strengthened by the discovery of the so-called "Fermi bubbles". These are two gamma-ray emitting bubbles located below and above the GC and extending as much as $10 \mathrm{kpc}$ 11, 12] which can be explained either in terms of AGN outflows [13, 14, 15], star capture events in the last $~ 10$ Myrs [16] or a nuclear starburst [12]. The observed gamma-ray activity fits the picture that until very recently $\operatorname{SgrA}^{*}$ was orders of magnitude more luminous in X-ray than it currently is [17]. If the bubble is caused by an AGN, the kinematics is consistent with an outburst which lasts $0.1-1 \mathrm{Myr}$ [13].

It is difficult to infer the corresponding bolometric luminosity, and the current estimation of the peak luminosity falls in the range of about $(3-100) \%$ of the Eddington limit $L_{\mathrm{Edd}} \simeq 5 \times 10^{44} \mathrm{erg} \mathrm{s}^{-1}[\underline{6}$, 18], which is a usual value for an AGN. This level of activity fits the framework of the aforementioned stochastic AGN model.

Current observations show that AGNs radiating at a luminosity close to the Eddington limit emit about $1 \%$ of their bolometric luminosity in hard X-ray $(h \nu>2 \mathrm{keV})[19,20]$. Theoretically, we can explain this in the context of a hot corona screening the accretion disc, as put forward by [21, 22, 23]. This means that the past activity of the MW must have had a relatively important hard X-ray component.

The implications are interesting because of the potential impact that this hard X-ray could have had on Earth in the past. We note that this idea is not new. Indeed, 24, 25, 26] looked into this possibility but did not take into account that this might have happened much more recently than they thought, because they lacked the observational evidence and theoretical framework.

We note that the consequences of an enhanced hard X-ray flux have been studied in detail in a different scenario: that of a near-by gamma-ray burst [27, 28]. The authors claimed that a sudden enhancement of this irradiation on Earth could increase the ionization of the upper atmosphere, leading to a chain of secondary reactions, among which ozone depletion, to a degree which depends on the fluence.

The magnitude of the impact in this or any other scenario is determined by 
the intensity and duration of the X-ray irradiation, as well as the spectral shape of the ionizing radiation. In this article we address these factors in the scenario that we propose: A possible recent active past of SgrA* .

\section{X-ray irradiation from Sagittarius $A^{*}$}

In this section we consider two different possibilities for the outburst of radiation originating at the Galactic Centre: A relatively recent AGN-like episode and a stellar tidal disruption event in a quiescent $\mathrm{SgrA}^{*}$. We compare the Xray irradiation levels in both scenarios with those originating from three degrees of current solar activity: a maximum, a minimum and a strong solar flare.

\subsection{A recent AGN-like episode}

We can derive the X-ray irradiation that potentially hit Earth in the past by using the model of [19] for a sample of unobscured AGNs. The unabsorbed, or unobscured, X-ray spectral energy distribution (SED, i.e. the luminosity per logarithmic energy bin) of an AGN can be modeled with a power law, which is consistent with the corona model, as discussed in the introduction.

The model of [19] provides us with the normalization and power-law index. According to these templates, given the ratio $\lambda_{\text {Edd }} \equiv L_{\text {bol }} / L_{\text {Edd }}$ between the bolometric luminosity $L_{\mathrm{bol}}$ and the Eddington luminosity $L_{\mathrm{Edd}}$, the luminosity $L_{2-10 \mathrm{keV}}$ of the photons in the band $2 \mathrm{keV}<h \nu<10 \mathrm{keV}$ scales as

$$
\log \left(L_{2-10 \mathrm{keV}} / L_{\mathrm{bol}}\right)=-0.773 \log \left(\lambda_{\mathrm{Edd}}\right)-2.004 .
$$

The photon index $\Gamma$, the index of a power law which characterizes the number of photons in each energy bin, is given by

$$
\Gamma=0.564 \log \left(\lambda_{\text {Edd }}\right)+2.246 \text {. }
$$

The SED will have a power-law index of $-\Gamma+2$. When converting the SED to the spectral irradiance on Earth $\left(\nu F_{\nu}\right)$, we apply the extinction law of the Galactic plane $\left(A_{K}=7.0\right.$ and $\left.R_{V}=3.1\right)$, following [29]. We do not consider 
the radiation from the accretion disc because, given the Eddington ratio of our interest $\left(\lambda_{\mathrm{Edd}}>0.1\right)$, the disc SED peaks at UV and soft X-ray bands, where the photons will be completely absorbed due to the high extinction. I.e. the irradiation comes mostly from the corona and we neglect the disc.

Having derived the X-ray flux as a function of photon energy on Earth originating from a past violent outburst in $\operatorname{SgrA}^{*}$, we compare it to current levels from the Sun, as provided by [30], in their Figure 6. We depict the comparison in Figure 1.

We compare our analytical fitting with detailed, numerical simulations of an accretion disc including the corona structure. The numerical simulations follow the model of [31]. We adopt different values of the ratio of the sum of gas pressure and radiation pressure to the magnetic pressure in the disc, $\beta$, of 50 and 100, and different values for the $\alpha$ viscosity of the disc, of 0.1 and 0.3 . For the range of accretion values that we use in this work, we find no significant difference between the empirical flux and the numerical simulations, so that for convenience we use the former.

The most important features from Figure 1 are that: (i) At about $h \nu>2$ $\mathrm{keV}$ the irradiance originating from an active past of $\mathrm{SgrA}^{*}$ exceeds that from the Sun during the solar minimum, regardless of $\lambda_{\text {Edd }}$. (ii) Starting at about $20-30 \mathrm{keV}$, the SED from the assumption that SgrA* was active in the past starts to become higher than that from the highest value which we can expect from the Sun, a strong X-class solar flare. (iii) At around $h \nu>10 \mathrm{keV}$ the relation between irradiance and $\lambda_{\text {Edd }}$ changes, meaning that lower values of accretion result in an even higher irradiance. This has already been explained in the disc corona model [21, 22]. (iv) In the assumption that $\mathrm{SgrA}^{*}$ was active in the past, the SED extends out to higher frequencies than what we can expect from the irrandiance of the Sun, up to $h \nu \sim 10^{2} \mathrm{keV}[20]$, the band of soft $\gamma$-ray. This makes the potential active episode of SgrA* brighter than the Sun in the hard-X-ray/soft- $\gamma$-ray, for all possible solar scenarios. 


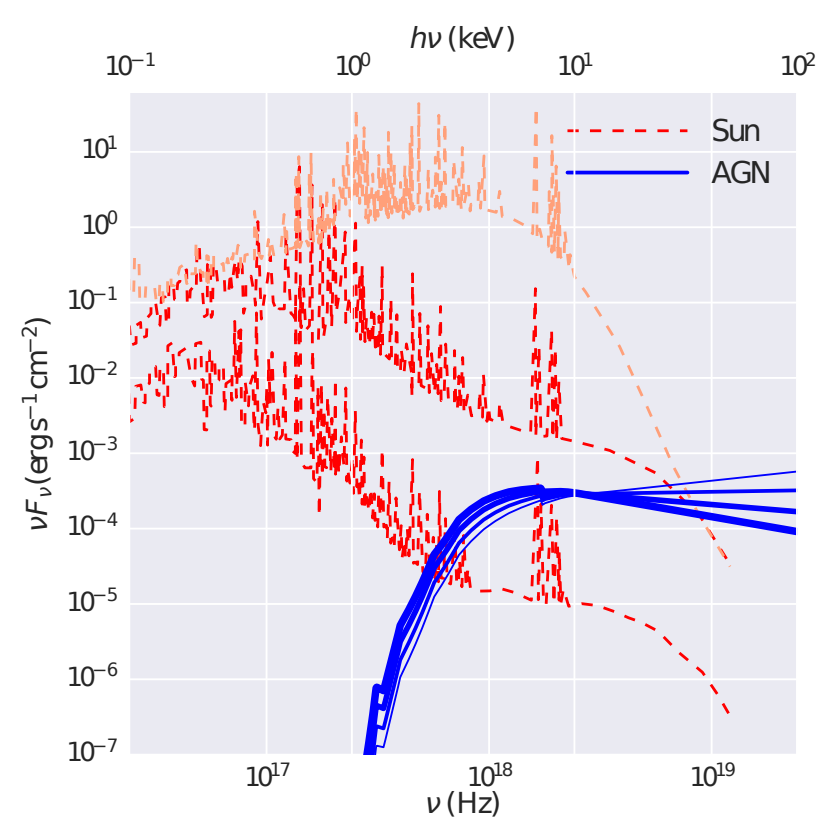

Figure 1: Comparison of X-ray irradiance on Earth from the Sun today (the dashed lines) and a potential AGN-like episode (the four solid lines) originating at the Galactic Centre. The thickness of the solid lines corresponds to different accretion rates, more specifically to $\lambda_{\text {Edd }}=(0.1,0.3,1,3)$, respectively (thicker means more efficient accretion). The dashed lines, taken from [30], from the bottom to the top correspond to a minimum irradiance during the solar cycle, a maximum one, and the top-most corresponds to an intense X-class solar flare. 


\subsection{A tidal disruption event}

The scenario that we consider now is the following: The X-ray luminosity of SgrA* can be temporarily enhanced by a tidal disruption of a star close to the SMBH [32, 33]. The event rate is of about once every $10^{4}-10^{5}$ years during the quiescent phase of $\operatorname{SgrA}^{*}[34]$.

Historically, we know that TDEs must have a (1) soft, thermal SED with an effective temperature of $k T \simeq 0.1 \mathrm{keV}$, as already put forward by the pioneering theoretical work of [32], but see also [35, 36, 37] for more recent works. Indeed, this soft component has been observed in TDE candidates [33].

However, as we started harvesting more and more data, observations have revealed (2) a hard X-ray emission from some TDE candidates [38, 39, 40]. These TDE candidates exhibit strong power-law components with $2<\Gamma<5$ in the energy band $h \nu>2 \mathrm{keV}$. This hard power-law component has an exponent of $\Gamma=3$ whose luminosity is $10 \%$ of the black-body emission, as empirically fitted by [41]

A possible theoretical explanation could be that these originate from shockheated, incompletely-thermalized material around the SMBH [35, 41]. In these models, the emission would be isotropic. We note here that we have observed hard X-ray irradiances exceeding by orders of magnitude the maximum what can be expected from these models [42, 43, 44, 45]. In these cases, a highly collimated jet originating from the TDE could be the source [46, 47], but the chances that it is pointing exactly towards us should be very low. Additionally, the fraction of TDEs producing a jet should also be negligible [48]. For the rest of this article, we will hence not consider the jet model.

In Figure 2 we compare the irradiances of the two separated scenarios: The AGN-like and the TDE one. These TDE candidates exhibit strong power-law components with $2<\Gamma<5$ in the energy band $h \nu>2 \mathrm{keV}$. Observationally we lack the cadence to catch the peak of the event, which should happen during the first two weeks following the TDE.

Theoretically, the expected accretion during that time is so high, $L_{\mathrm{bol}}>$ $3 L_{\mathrm{Edd}}$ according to our model, even approaching $10^{2} L_{\mathrm{Edd}}$ in the first couple of 


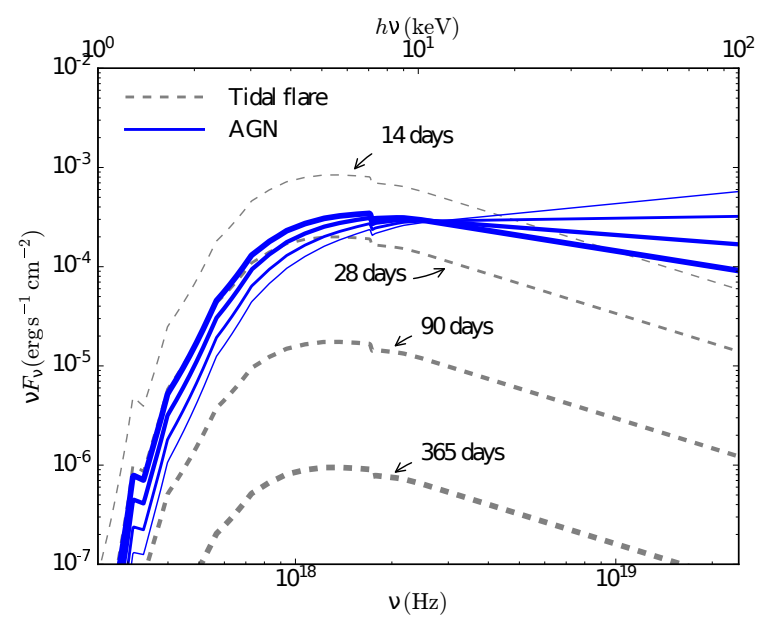

Figure 2: Spectral irradiances from the AGN-like and TDE scenarios. The four solid lines with increasing thickness depict the irradiances of the tidal disruption $(14,28,90,365)$ days after the disruption of a solar-type star. The four dashed curves are for the AGN-like possibility, adopted from Figure 1. We apply the extinction law on the irradiance [29], so that the soft component is completely absorbed and hence does not show in the figure.

days, that there is not a well-established consensus on our current accretion-disc models for it (see, for instance, 35, 41, 49, 50, 51]). I.e. we cannot convert the fallback rate of stellar debris into luminosity. This is why we only display from day 14 onward in our figure.

In the figure we see that the TDE and the AGN-like models seem to be at similar levels in the hard X-ray band, at least for a period of time not superior to one month. However, we note that the structure of the star (its mass, and radius, mostly), as well as the orbital features of the TDE can lead to significant changes in the flux and timescales (see e.g. equation 13 in [37]).

\section{Fluxes and fluences}

In the previous section we have seen that the integrated hard (i.e. starting at $\sim 2 \mathrm{keV}$ ) X-ray flux is of about $10^{-3} \mathrm{erg} \mathrm{s}^{-1} \mathrm{~cm}^{-2}$, comparable to that from the active sun. This is true for the AGN-like scenario and also for the TDE one, 
but only for the first two weeks, since after that period of time the flux drops significantly, up to three orders of magnitude after the first year.

Besides irradiance, another quantity which is often used to evaluate the lethality of ionizing radiation is the so-called fluence, i.e. the total amount of radiation injected into one unit area. For this work, we consider fluence to be the total integrated emitted energy in hard X-ray. We find that it is $10^{3} \mathrm{erg} \mathrm{cm}^{-2}$ for the TDE scenario, being conservative, since we only consider a week of duration after the first two weeks. The AGN-like case yields $3 \times 10^{9} \mathrm{erg} \mathrm{cm}^{-2}$ where we have assumed a total duration of $10^{5}$ years, to be consistent with the constraint from the kinematics of the Fermi bubble [13].

The impact of hard X-ray flux and fluence on Earth has been discussed in the related literature in the context of gamma-ray bursts (GRBs):

(i) Flux: It has been shown by [52, 53, 54] that in the past 30 years several distant (soft) gamma-ray bursts (GRBs) had induced similar level of irradiation on Earth, with fluxes of about $10^{-3} \mathrm{erg} \mathrm{s}^{-1} \mathrm{~cm}^{-2}$ at $h \nu>5 \mathrm{keV}$, causing disturbances of the ionosphere. The kind of flux that we consider in the AGN- and TDE-like scenarios could hence potentially lead to a more serious disturbance of the ionosphere, because they last longer, ranging from a few weeks to as long as $10^{5}$ years.

(ii) Fluence: This is why probably it is more useful to look not at the flux, but at the fluence - Possible nearby supernova numerical studies predict a hard X-ray fluence in the range $(2-3) \times 10^{6} \mathrm{erg} \mathrm{cm}^{-2}$, which leads to an ozone depletion of about $3 \%$ 28]. Fluences of about $10^{8} \mathrm{erg} \mathrm{cm}^{-2}$ lead to removal factors as high as $(20-30) \%$ [55]. While the TDE-like case is unlikely to reach these levels, as we have seen, the AGN-like case exceeds this number by one order of magnitude.

\section{Discussion}

There is growing evidence that $\mathrm{SgrA}^{*}$ must have been active in the past, more specifically as recently as several Myr ago. This fact has motivated us to 


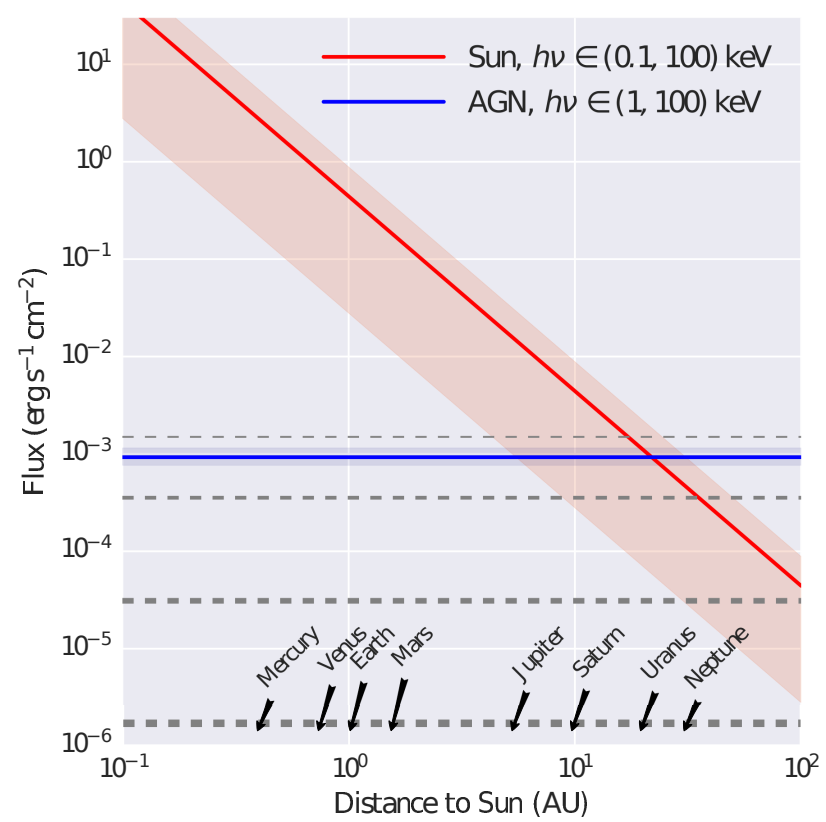

Figure 3: X-ray irradiances across the solar system for the TDE and AGN-like scenarios. The TDE is shown as grey, dashed lines, following the same code as in Fig. (2) for the time duration. The AGN maximum and minimum correspond to the upper and lower boundaries of the blue-shaded area, respectively, and the thick blue line the average. The solar irradiance is displayed as a red-shaded area. The upper- and lower limits correspond to the solar maximum and minimum, respectively, and the thick red line the average of the two. We mark the position of the solar planets.

study the irradiation from a recent active episode of $\mathrm{SgrA}^{*}$ on Earth.

In this work we have estimated the flux on Earth emitted by such as an episode. In particular, we have considered two different possibilities, a TDE and a recent AGN-like activity. We show that for Earth, and out to Jupiter, the flux from a violent $\mathrm{X}$-ray activity originating at $\mathrm{SgrA}^{*}$ is negligible compared to the sun's. I.e., from about $10 \mathrm{AU}$ to the edge of the solar system, the irradiation from $\mathrm{SgrA}^{*}$ during its outburst predominates in the entire X-ray band of $h \nu>1$ $\mathrm{keV}$. Most of the gas giants in the solar system, including their moons, lie in this region, but the corresponding reaction of their atmosphere has not been explored. 
X-ray irradiation can also drive the chemical reactions in dense molecular clouds [56], and enhance the abundance of organic molecules in protoplanetary discs [57]. The implication is that the ancient outbursts from SgrA* may have played an important role in shaping the habitable environments on planets, especially on those exoplanets which are much closer to the Galactic Centre than our solar system is.

\section{Acknowledgements}

We thank Fukun Liu for his suggestion of using empirical SED templates, and Ran Wang for providing references for SEDs. We thank Erlin Qiao and Jieying Liu for the dedicated numerical simulations for the X-ray spectrum. We are grateful to Emily Davidson for proofreading our manuscript. This work was supported by the National Key R\&D Program of China (2016YFA0400702) and the National Science Foundation of China (11721303). Pau Amaro Seoane thanks Marta Masini for her outstanding and unique assistance and endurance during the last days in the preparation of the manuscript in Beijing. He acknowledges support from the Ramón y Cajal Programme of the Ministry of Economy, Industry and Competitiveness of Spain, as well as the COST Action GWverse CA16104.

\section{References}

\section{References}

[1] J. Kormendy, L. C. Ho, Coevolution (Or Not) of Supermassive Black Holes and Host Galaxies, araa 51 (2013) 511-653. arXiv:1304.7762, doi:10.1146/annurev-astro-082708-101811.

[2] R. Genzel, F. Eisenhauer, S. Gillessen, The Galactic Center massive black hole and nuclear star cluster, Reviews of Modern Physics 82 (2010) 31213195. arXiv:1006.0064, doi:10.1103/RevModPhys.82.3121. 
[3] G. Gilmore, R. F. G. Wyse, J. E. Norris, Deciphering the Last Major Invasion of the Milky Way, apjl 574 (2002) L39-L42. arXiv:astro-ph/0207106, doi:10.1086/342363.

[4] P. F. Hopkins, L. Hernquist, Fueling Low-Level AGN Activity through Stochastic Accretion of Cold Gas, apjs 166 (2006) 1-36. arXiv:astro-ph/0603180, doi:10.1086/505753.

[5] P. G. Mezger, W. J. Duschl, R. Zylka, The Galactic Center: a laboratory for AGN?, The Astron. Astrophys. Rev. 7 (1996) 289-388. doi:10.1007/s001590050007.

[6] S. Nayakshin, J. Cuadra, A self-gravitating accretion disk in Sgr A* a few million years ago: Is Sgr A* a failed quasar? 437 (2005) 437-445. arXiv:astro-ph/0409541, doi:10.1051/0004-6361:20042052,

[7] J. Bland-Hawthorn, P. R. Maloney, R. S. Sutherland, G. J. Madsen, Fossil Imprint of a Powerful Flare at the Galactic Center along the Magellanic Stream, ApJ 778 (2013) 58. arXiv:1309.5455, doi:10.1088/0004-637X/778/1/58

[8] P. Amaro-Seoane, X. Chen, The Fragmenting Past of the Disk at the Galactic Center: The Culprit for the Missing Red Giants, ApJ Lett. 781 (2014) L18. arXiv:1310.0458, doi:10.1088/2041-8205/781/1/L18.

[9] X. Chen, P. Amaro-Seoane, A Rapidly Evolving Region in the Galactic Center: Why S-stars Thermalize and More Massive Stars are Missing, ApJ Lett. 786 (2014) L14. arXiv:1401.6456, doi:10.1088/2041-8205/786/2/L14

[10] X. Chen, P. Amaro-Seoane, Sculpting the stellar cusp in the galactic center, Classical and Quantum Gravity 32 (6) (2015) 064001. arXiv:1410.3824, doi:10.1088/0264-9381/32/6/064001. 
[11] G. Dobler, D. P. Finkbeiner, I. Cholis, T. Slatyer, N. Weiner, The Fermi Haze: A Gamma-ray Counterpart to the Microwave Haze, ApJ 717 (2010) 825-842. arXiv:0910.4583, doi:10.1088/0004-637X/717/2/825.

[12] M. Su, T. R. Slatyer, D. P. Finkbeiner, Giant Gamma-ray Bubbles from Fermi-LAT: Active Galactic Nucleus Activity or Bipolar Galactic Wind? 724 (2010) 1044-1082. arXiv:1005.5480, doi: $10.1088 / 0004-637 \mathrm{X} / 724 / 2 / 1044$

[13] K. Zubovas, A. R. King, S. Nayakshin, The Milky Way's Fermi bubbles: echoes of the last quasar outburst?, MNRAS 415 (2011) L21-L25. arXiv:1104.5443, doi:10.1111/j.1745-3933.2011.01070.x.

[14] F. Guo, W. G. Mathews, The Fermi Bubbles. I. Possible Evidence for Recent AGN Jet Activity in the Galaxy, ApJ 756 (2012) 181. arXiv:1103.0055, doi:10.1088/0004-637X/756/2/181.

[15] G. Mou, F. Yuan, D. Bu, M. Sun, M. Su, Fermi Bubbles Inflated by Winds Launched from the Hot Accretion Flow in Sgr A*, ApJ 790 (2014) 109. arXiv:1403.2129, doi:10.1088/0004-637X/790/2/109,

[16] K.-S. Cheng, D. O. Chernyshov, V. A. Dogiel, C.-M. Ko, W.-H. Ip, Origin of the Fermi Bubble, ApJ Lett. 731 (2011) L17. arXiv:1103.1002, doi:10.1088/2041-8205/731/1/L17

[17] G. Ponti, M. R. Morris, R. Terrier, A. Goldwurm, Traces of Past Activity in the Galactic Centre, in: D. F. Torres, O. Reimer (Eds.), Cosmic Rays in Star-Forming Environments, Vol. 34 of Astrophysics and Space Science Proceedings, 2013, p. 331. arXiv:1210.3034, doi:10.1007/978-3-642-35410-6\_26.

[18] J. Bland-Hawthorn, P. R. Maloney, R. S. Sutherland, G. J. Madsen, Fossil Imprint of a Powerful Flare at the Galactic Center along the Magellanic Stream 778 (2013) 58. arXiv:1309.5455, doi: $10.1088 / 0004-637 X / 778 / 1 / 58$ 
[19] C. Jin, M. Ward, C. Done, A combined optical and X-ray study of unobscured type 1 active galactic nuclei - III. Broadband SED properties 425 (2012) 907-929. arXiv:1109.2069 doi:10.1111/j.1365-2966.2012.21272.x.

[20] R. V. Vasudevan, R. F. Mushotzky, C. S. Reynolds, A. C. Fabian, A. M. Lohfink, A. Zoghbi, L. C. Gallo, D. Walton, The Hard X-Ray Perspective on the Soft X-Ray Excess 785 (2014) 30. arXiv:1402.3591, doi:10.1088/0004-637X/785/1/30

[21] Y. Lu, Q. Yu, Two Different Accretion Classes in Seyfert 1 Galaxies and QSOS, apjl 526 (1999) L5-L8. arXiv:astro-ph/9911289, doi: $10.1086 / 312358$

[22] J.-M. Wang, K.-Y. Watarai, S. Mineshige, The Hot Disk Corona and Magnetic Turbulence in Radio-quiet Active Galactic Nuclei: Observational Constraints, apjl 607 (2004) L107-L110. arXiv: astro-ph/0407160, doi:10.1086/421906

[23] E. Qiao, B. F. Liu, The condensation of the corona for the correlation between the hard X-ray photon index $\Gamma$ and the reflection scaling factor real in active galactic nuclei, MNRAsarXiv:1701.04211, doi:10.1093/mnras/stx121.

[24] J. N. Clarke, Extraterrestrial intelligence and galactic nuclear activity, Icarus 46 (1981) 94-96. doi:10.1016/0019-1035(81)90078-6

[25] P. A. Laviolette, Cosmic-ray volleys from the Galactic Center and their recent impact on the earth environment, Earth Moon and Planets 37 (1987) 241-286. doi:10.1007/BF00116639

[26] G. Gonzalez, Habitable Zones in the Universe, Origins of Life and Evolution of the Biosphere 35 (2005) 555-606. arXiv:astro-ph/0503298, doi:10.1007/s11084-005-5010-8. 
[27] L. R. Dartnell, Ionizing Radiation and Life, Astrobiology 11 (2011) 551582. doi:10.1089/ast.2010.0528.

[28] A. L. Melott, B. C. Thomas, Astrophysical Ionizing Radiation and Earth: A Brief Review and Census of Intermittent Intense Sources, Astrobiology 11 (2011) 343-361. arXiv:1102.2830, doi:10.1089/ast.2010.0603.

[29] J. C. Tan, B. T. Draine, X-Ray Scattering Halos from the Galactic Center: Implications for Diffuse Emission around Sagittarius A* 606 (2004) 296305. arXiv:astro-ph/0310442, doi:10.1086/382865

[30] G. Peres, S. Orlando, F. Reale, R. Rosner, H. Hudson, The Sun as an X-Ray Star. II. Using theYohkoh/Soft X-Ray Telescope-derived Solar Emission Measure versus Temperature to Interpret Stellar X-Ray Observations 528 (2000) 537-551. doi:10.1086/308136.

[31] J. Y. Liu, E. L. Qiao, B. F. Liu, Revisiting the Structure and Spectrum of the Magnetic-reconnection-heated Corona in Luminous AGNs, ApJ 833 (2016) 35. arXiv:1611.07149, doi:10.3847/1538-4357/833/1/35

[32] M. J. Rees, Tidal disruption of stars by black holes of 10 to the 6th10 to the 8th solar masses in nearby galaxies 333 (1988) 523-528. doi:10.1038/333523a0,

[33] S. Komossa, Tidal disruption of stars by supermassive black holes: The X-ray view, in: European Physical Journal Web of Conferences, Vol. 39 of European Physical Journal Web of Conferences, 2012, p. 2001. doi:10.1051/epjconf/20123902001.

[34] D. Merritt, The Distribution of Stars and Stellar Remnants at the Galactic Center 718 (2010) 739-761. arXiv:0909.1318, doi: $10.1088 / 0004-637 \mathrm{X} / 718 / 2 / 739$

[35] A. Ulmer, Flares from the Tidal Disruption of Stars by Massive Black Holes 514 (1999) 180-187. doi:10.1086/306909. 
[36] L.-X. Li, R. Narayan, K. Menou, The Giant X-Ray Flare of NGC 5905: Tidal Disruption of a Star, a Brown Dwarf, or a Planet? 576 (2002) 753761. arXiv:astro-ph/0203191, doi:10.1086/341890,

[37] X. Chen, F. K. Liu, Is There an Intermediate Massive Black Hole in the Galactic Center: Imprints on the Stellar Tidal-disruption Rate 762 (2013) 95. arXiv:1211.4609, doi:10.1088/0004-637X/762/2/95

[38] D. Lin, E. R. Carrasco, D. Grupe, N. A. Webb, D. Barret, S. A. Farrell, Discovery of an Ultrasoft X-Ray Transient Source in the 2XMM Catalog: A Tidal Disruption Event Candidate 738 (2011) 52. arXiv:1106.0744, doi: $10.1088 / 0004-637 X / 738 / 1 / 52$

[39] R. D. Saxton, A. M. Read, P. Esquej, S. Komossa, S. Dougherty, P. Rodriguez-Pascual, D. Barrado, A tidal disruption-like X-ray flare from the quiescent galaxy SDSS J120136.02+300305.5 541 (2012) A106. arXiv:1202.5900, doi:10.1051/0004-6361/201118367.

[40] M. Nikołajuk, R. Walter, Tidal disruption of a super-Jupiter by a massive black hole 552 (2013) A75. arXiv:1304.0397, doi:10.1051/0004-6361/201220664

[41] L. E. Strubbe, E. Quataert, Spectroscopic signatures of the tidal disruption of stars by massive black holes 415 (2011) 168-180. arXiv:1008.4131, doi:10.1111/j.1365-2966.2011.18686.x.

[42] D. N. Burrows, J. A. Kennea, G. Ghisellini, V. Mangano, B. Zhang, K. L. Page, M. Eracleous, P. Romano, T. Sakamoto, A. D. Falcone, J. P. Osborne, S. Campana, A. P. Beardmore, A. A. Breeveld, M. M. Chester, R. Corbet, S. Covino, J. R. Cummings, P. D'Avanzo, V. D'Elia, P. Esposito, P. A. Evans, D. Fugazza, J. M. Gelbord, K. Hiroi, S. T. Holland, K. Y. Huang, M. Im, G. Israel, Y. Jeon, Y.-B. Jeon, H. D. Jun, N. Kawai, J. H. Kim, H. A. Krimm, F. E. Marshall, P. Mészáros, H. Negoro, N. Omodei, W.-K. Park, J. S. Perkins, M. Sugizaki, H.-I. Sung, 
G. Tagliaferri, E. Troja, Y. Ueda, Y. Urata, R. Usui, L. A. Antonelli, S. D. Barthelmy, G. Cusumano, P. Giommi, A. Melandri, M. Perri, J. L. Racusin, B. Sbarufatti, M. H. Siegel, N. Gehrels, Relativistic jet activity from the tidal disruption of a star by a massive black hole, Nat 476 (2011) 421-424. arXiv:1104.4787, doi:10.1038/nature10374,

[43] A. J. Levan, N. R. Tanvir, S. B. Cenko, D. A. Perley, K. Wiersema, J. S. Bloom, A. S. Fruchter, A. d. U. Postigo, P. T. O'Brien, N. Butler, A. J. van der Horst, G. Leloudas, A. N. Morgan, K. Misra, G. C. Bower, J. Farihi, R. L. Tunnicliffe, M. Modjaz, J. M. Silverman, J. Hjorth, C. Thöne, A. Cucchiara, J. M. C. Cerón, A. J. Castro-Tirado, J. A. Arnold, M. Bremer, J. P. Brodie, T. Carroll, M. C. Cooper, P. A. Curran, R. M. Cutri, J. Ehle, D. Forbes, J. Fynbo, J. Gorosabel, J. Graham, D. I. Hoffman, S. Guziy, P. Jakobsson, A. Kamble, T. Kerr, M. M. Kasliwal, C. Kouveliotou, D. Kocevski, N. M. Law, P. E. Nugent, E. O. Ofek, D. Poznanski, R. M. Quimby, E. Rol, A. J. Romanowsky, R. Sánchez-Ramírez, S. Schulze, N. Singh, L. van Spaandonk, R. L. C. Starling, R. G. Strom, J. C. Tello, O. Vaduvescu, P. J. Wheatley, R. A. M. J. Wijers, J. M. Winters, D. Xu, An Extremely Luminous Panchromatic Outburst from the Nucleus of a Distant Galaxy, Science 333 (2011) 199. arXiv:1104.3356, doi:10.1126/science.1207143.

[44] S. B. Cenko, H. A. Krimm, A. Horesh, A. Rau, D. A. Frail, J. A. Kennea, A. J. Levan, S. T. Holland, N. R. Butler, R. M. Quimby, J. S. Bloom, A. V. Filippenko, A. Gal-Yam, J. Greiner, S. R. Kulkarni, E. O. Ofek, F. Olivares E., P. Schady, J. M. Silverman, N. R. Tanvir, D. Xu, Swift J2058.4+0516: Discovery of a Possible Second Relativistic Tidal Disruption Flare?, ApJ 753 (2012) 77. arXiv:1107.5307, doi:10.1088/0004-637X/753/1/77.

[45] G. C. Brown, A. J. Levan, E. R. Stanway, N. R. Tanvir, S. B. Cenko, E. Berger, R. Chornock, A. Cucchiaria, Swift J1112.2-8238: a candidate relativistic tidal disruption flare, MNRAS 452 (2015) 4297-4306. arXiv:1507.03582, doi:10.1093/mnras/stv1520. 
[46] J. S. Bloom, D. Giannios, B. D. Metzger, S. B. Cenko, D. A. Perley, N. R. Butler, N. R. Tanvir, A. J. Levan, P. T. O'Brien, L. E. Strubbe, F. De Colle, E. Ramirez-Ruiz, W. H. Lee, S. Nayakshin, E. Quataert, A. R. King, A. Cucchiara, J. Guillochon, G. C. Bower, A. S. Fruchter, A. N. Morgan, A. J. van der Horst, A Possible Relativistic Jetted Outburst from a Massive Black Hole Fed by a Tidally Disrupted Star, Science 333 (2011) 203. arXiv:1104.3257, doi:10.1126/science.1207150,

[47] B. A. Zauderer, E. Berger, A. M. Soderberg, A. Loeb, R. Narayan, D. A. Frail, G. R. Petitpas, A. Brunthaler, R. Chornock, J. M. Carpenter, G. G. Pooley, K. Mooley, S. R. Kulkarni, R. Margutti, D. B. Fox, E. Nakar, N. A. Patel, N. H. Volgenau, T. L. Culverhouse, M. F. Bietenholz, M. P. Rupen, W. Max-Moerbeck, A. C. S. Readhead, J. Richards, M. Shepherd, S. Storm, C. L. H. Hull, Birth of a relativistic outflow in the unusual $\gamma$-ray transient Swift J164449.3+573451, Nat 476 (2011) 425-428. arXiv:1106.3568, doi:10.1038/nature10366

[48] F. De Colle, J. Guillochon, J. Naiman, E. Ramirez-Ruiz, The Dynamics, Appearance, and Demographics of Relativistic Jets Triggered by Tidal Disruption of Stars in Quiescent Supermassive Black Holes, ApJ 760 (2012) 103. arXiv:1205.1507, doi:10.1088/0004-637X/760/2/103,

[49] E. R. Coughlin, M. C. Begelman, Hyperaccretion during Tidal Disruption Events: Weakly Bound Debris Envelopes and Jets 781 (2014) 82. arXiv:1312.5314, doi:10.1088/0004-637X/781/2/82.

[50] T. Piran, A. Sạdowski, A. Tchekhovskoy, Jet and disc luminosities in tidal disruption events, MNRAS 453 (2015) 157-165. arXiv:1501.02015, doi:10.1093/mnras/stv1547.

[51] M. C. Begelman, M. Volonteri, Hyperaccreting black holes in galactic nuclei, MNRAS 464 (2017) 1102-1107. arXiv:1609.07137, doi:10.1093/mnras/stw2446 
[52] G. J. Fishman, U. S. Inan, Observation of an ionospheric disturbance caused by a gamma-ray burst 331 (1988) 418-420. doi:10.1038/331418a0.

[53] U. S. Inan, N. G. Lehtinen, S. J. Lev-Tov, M. P. Johnson, T. F. Bell, K. Hurley, Ionization of the lower ionosphere by $\gamma$-rays from a Magnetar: Detection of a low energy (3-10 keV) component, Geophysical Research Letters 26 (1999) 3357-3360. doi:10.1029/1999GL010690.

[54] M. Mandea, G. Balasis, FAST TRACK PAPER: The SGR 180620 magnetar signature on the Earth's magnetic field, Geophysical Journal International 167 (2006) 586-591. arXiv:0710.2793, doi:10.1111/j.1365-246X.2006.03125.x.

[55] L. M. Ejzak, A. L. Melott, M. V. Medvedev, B. C. Thomas, Terrestrial Consequences of Spectral and Temporal Variability in Ionizing Photon Events 654 (2007) 373-384. arXiv:astro-ph/0604556, doi:10.1086/509106.

[56] J. H. Krolik, T. R. Kallman, X-ray ionization and the Orion molecular cloud 267 (1983) 610-624. doi:10.1086/160897.

[57] J. K. Teske, J. R. Najita, J. S. Carr, I. Pascucci, D. Apai, T. Henning, Measuring Organic Molecular Emission in Disks with Lowresolution Spitzer Spectroscopy 734 (2011) 27. arXiv:1104.0249, doi:10.1088/0004-637X/734/1/27. 